\title{
GERD-Related Health Care Utilization, Therapy, and Reasons for Transfer of GERD Patients Between Primary Care Providers and Gastroenterologists in a US Managed Care Setting
}

\author{
Rachel Halpern · Smita Kothari · Mahesh Fuldeore • \\ Victoria Zarotsky • Victoria Porter • Omar Dabbous • \\ Jay L. Goldstein
}

Received: 6 February 2009/ Accepted: 16 July 2009/Published online: 21 August 2009

(C) Springer Science+Business Media, LLC 2009

\begin{abstract}
Purpose Patient flow between primary care physicians and gastroenterologists in the continuum of gastroesophageal reflux disease (GERD) care is poorly understood. Using administrative claims data from a large US health plan linked with data abstracted from medical records, we examined: health care resource utilization for GERD subjects treated by primary care physicians (PCPs) and gastroenterologists (GEs), determinants of GERD subject transfer between these physician types, and reasons for GERD therapy change.

Results Within a sample of 169,884 patients, 211,043 PCP-based episodes of care and 40,304 GE-based episodes of care were developed. In unadjusted comparisons, GE episodes were characterized by more endoscopic procedures, on average (50.5/100 episodes), compared with PCP episodes $(6.3 / 100, \quad P<0.001)$. Multivariate analysis showed that patients with esophagitis had $57.3 \%$ higher odds $(P<0.01)$ of transfer from PCP to GE compared with
\end{abstract}

R. Halpern · V. Zarotsky · V. Porter

Health Economics and Outcomes Research, i3 Innovus,

Eden Prairie, MN, USA

S. Kothari · M. Fuldeore

Health Economics and Outcomes Research, Formerly of TAP

Pharmaceutical Products, Inc., Lake Forest, IL, USA

O. Dabbous

Health Economics and Outcomes Research, Takeda

Pharmaceuticals North America, Inc., Deerfield, IL, USA

\section{J. L. Goldstein ( $₫)$}

Department of Medicine, University of Illinois at Chicago,

840 South Wood Street (m/c787), Room 1020, 10th Floor,

Chicago, IL 60612, USA

e-mail: jlgoldst@uic.edu patients without esophagitis; patients with esophageal stricture had $98.6 \%$ higher odds $(P<0.01)$ of PCP-GE transfer. Patients with endoscopy during a first GE episode had $32.2 \%$ higher odds of transfer to a PCP $(P<0.01)$. The principal reasons for change in GERD therapy were no change or worsening of symptoms $(51.7 \%$ of PCP charts; $9.5 \%$ of GE charts) and lack of response to therapy $(51.7 \%$ of PCP charts, $26.2 \%$ of GE charts).

Conclusion Resource utilization varies greatly based on the physician's specialty. We infer that timely transfer of GERD patients to gastroenterologists when empiric treatment is insufficient may lead to more efficient clinical management.

Keywords Gastroesophageal reflux ·

Primary health care · Gastroenterology ·

Physician's practice patterns · Health services .

Medication therapy management

\section{Introduction}

Gastroesophageal reflux disease (GERD) is a common chronic condition associated with significant morbidity, resource consumption, and cost. An estimated 44\% of the US adult population has symptoms of heartburn at least once per month [1], and approximately $14 \%$ of Americans have gastroesophageal symptoms weekly, and $7 \%$ have symptoms daily [1-3]. The annual total (direct and indirect) cost associated with management of GERD is estimated at more than US $\$ 14$ billion in the USA, $60 \%$ of which is spent on medication [4].

Treatment for GERD is aimed at managing symptoms and preventing complications. According to American College of Gastroenterology (ACG) guidelines, the initial 
diagnosis of GERD is based on patient history, and empiric acid suppression therapy should be used for patients who have typical GERD symptoms without atypical manifestations, warning signs, or symptoms suggestive of complicated disease $[1,5]$. Patients who respond positively to treatment are presumed to have GERD. Further diagnostic testing is recommended for patients who do not respond to treatment, have symptoms (e.g., dysphagia, odynophagia, bleeding, anemia, weight loss) suggestive of complications or other conditions, or experience symptoms of sufficient duration to put them at risk for developing Barrett's esophagus [6]. Endoscopy, although it lacks sensitivity for identifying pathologic reflux, is the gold standard to assess esophageal complications of GERD [7]. While these recommendations and others exist in the literature, acceptance at the provider level and their use in clinical practice vary greatly [8]; this variability has significant impact on the cost of care.

Most of the data that provide insight into the ways that physicians approach GERD patients come from physician surveys. Patients with GERD symptoms frequently present to their primary care physicians (PCPs) initially [9, 10]. A commonly cited treatment strategy among PCPs is empiric therapy, often using a "step-up" approach beginning with antacids or H2-receptor antagonists (H2RAs) and progressing to proton pump inhibitors (PPIs) for patients who fail to respond to therapy or who have esophagitis [11], although results from some surveys show that PCPs may also employ a "step-down" approach, beginning with PPIs $[8,12]$. PCPs often refer patients with inferred or diagnosed GERD to gastroenterologists or other specialists for diagnostic endoscopy $[11,13]$, when symptoms are severe or persistent $[11,13]$, or when patients are unresponsive to therapy [8]. Gastroenterologists (GEs) tend to use a more resource-intensive management approach to GERD, performing diagnostic procedures before commencing a treatment regimen, particularly for patients whose symptoms are moderate or severe [14, 15]. In addition, gastroenterologists are uniformly likely to use a step-down therapy regimen $[12,14]$.

To our knowledge, there has not been a national, administrative claims-based examination of GERD treatment strategies, and the ways in which those treatment strategies differ between PCPs and GEs. Unsuccessful management of GERD symptoms has important implications for health care resource consumption, particularly if GERD symptoms become chronic. The objectives of this study were to: quantify the variation in health care resource utilization, including pharmacologic therapy, between PCPs and GEs treating subjects with GERD; identify the determinants of transfers of GERD subjects between PCPs and GEs; and ascertain the reasons for changes in GERD therapy regimens.

\section{Methods}

The data for this study came from two sources: retrospective medical and pharmacy claims and enrollment information from a large US health plan; and information abstracted from 200 patient medical charts. The health plan provides national coverage, with the greatest concentration of plan members in the Midwest and South US census regions. The health plan comprises primarily discounted fee-for-service independent practice associations. The administrative claims data were de-identified and compliant with the provisions of the Health Insurance Portability and Accountability Act of 1996. Medical chart data came from records of a subset of subjects represented in the claims-based data set who received care both from PCPs and GEs for GERD or GERD symptoms over the study period. The claims-based data for medical chart abstraction candidates were re-identified upon approval from a privacy board. Health care resource utilization was measured with administrative claims. Reasons for transfers of GERD subjects between PCPs and GEs, GERD therapies including over-the-counter (OTC) medications, and reasons for GERD therapy changes, which cannot be determined from the claims data, were measured from variables developed from the medical chart abstractions.

\section{Claims Data-Based Study Sample}

Subjects with claims-based evidence of GERD during the period May 1, 2001 through November 30, 2005 were included in the study population. The criteria for evidence of GERD were either: (1) $\geq 2$ medical claims with a primary or secondary International Classification of Diseases, 9th Revision, Clinical Modification (ICD-9-CM) diagnosis code for reflux esophagitis (530.11), esophageal reflux (530.81) or heartburn (787.1); or (2) $\geq 1$ medical claim with a primary or secondary ICD-9-CM 530.11, 530.81 or 787.1 and either (a) $\geq 1$ pharmacy claim for a PPI (esomeprazole, lansoprazole, omeprazole, pantoprazole, rabeprazole), an H2RA (cimetidine, famotidine, nizatidine, or ranitidine) or "other" GERD agent (bethanechol, metoclopramide, sucralfate) or (b) $\geq 1$ medical claim with a primary or secondary ICD-9-CM code for esophageal stricture (530.3) Barrett's esophagus (530.85), esophageal ulcer $(530.2 \times)$ or esophagitis $(530.1 \times)$. The date of the first relevant pharmacy or medical claim was defined as the index date. Subjects in the study population also met the following criteria: continuous enrollment in a commercial health plan with medical and pharmacy benefits for 6 months before and at least 1 year after the index date; no medical claims with diagnoses indicating esophageal problems that could cast doubt on a GERD diagnosis, e.g., esophageal burns, toxic effects of caustic or corrosive agents, effects of 
radiation $^{1}$; and no medical claims with a physician specialty code of GE for a 3-month period preceding the index date to ensure that subjects were not under the ongoing care of a GE at the beginning of their post-index periods.

\section{GERD-Related Episodes}

Subject-level episodes of GERD-related care were constructed from subjects' index dates through the end of their post-index date periods with Episode Treatment Groups ${ }^{\mathrm{TM}}$ (ETG) software developed by Symmetry Health Data Systems ${ }^{\circledR}$. ETGs use diagnosis codes, procedure codes, and National Drug Codes (NDCs) to formulate clinically homogenous episodes of care by disease condition. The predefined ETG episode categories within which GERDrelated care was identified were: infections of the stomach and esophagus, with comorbidity; infections of the stomach and esophagus, without comorbidity; inflammation of the esophagus, with surgery; and inflammation of the esophagus, without surgery. Episodes begin with medical claims from clinician-provided services associated with the evaluation, management or treatment (e.g., physician office visit, surgery) of a condition [16] and end when there is an absence of condition-related care for a minimum period of time; for certain chronic conditions, including those used for this study, this period of quiescence is 180 days. Subjects could have multiple episodes.

\section{Measures}

Subject demographic variables included age, gender, geographic region, "new-onset" GERD (no medical claims with GERD-related ICD-9-CM codes and no pharmacy claims for GERD therapies used to identify the study population, and no medical claims with a specialty code for GE for any condition, during 6-month pre-index date period). Health status variables, measured during the 6-month pre-index date period, were GERD severity and comorbid conditions. GERD severity was a categorical variable with three values - high, medium, and low-and was measured with a diagnosis-based algorithm that factored in complications of GERD, comorbidities associated with GERD, and other conditions that increase the difficulty of GERD management. ${ }^{2}$ Comorbid conditions were measured with Clinical Classification Software managed by the Agency for Healthcare Research and Quality [17].

GERD-related health care utilization variables were measured between the beginning and end of each GERD

\footnotetext{
${ }^{1}$ ICD-9-CM codes are available from the corresponding author upon request.

${ }^{2}$ See footnote 1 .
}

episode. GERD-related utilization was defined with the ICD-9-CM codes used to identify the study sample, plus ICD-9-CM codes for esophageal dyskinesia (530.5), 787.2 (dysphagia), 786.50 and 786.59 (chest pain) in the first or second position on the medical claim; these additional diagnosis codes were selected based on examination of all claims for all GERD-related ETG-based episodes for 45 subjects, and the input of a GE experienced in GERD treatment. GERD-related utilization variables were counts of: office visits [Current Procedural Terminology (CPT) codes 99201-99205, 99211-99215, 99241-99245]; endoscopic procedures (CPT 43200-43272, 0008T); esophageal repair and manipulation procedures (CPT 43280-43289, 43300-43425, 43450-43460, 43499, e.g., esophagogastric fundoplasty, esophagoplasty, esophagostomy, dilation of esophagus); additional diagnostic procedures (e.g., esophageal motility study, Bernstein test: CPT 91010-91040); and GERD therapy (PPI, H2RA, other GERD agent) fills. In addition, episodes with at least one medical claim with a diagnosis for esophageal ulcer, esophagitis, esophageal stricture or Barrett's esophagus in the first or second position on claim were identified.

All episodes were categorized as PCP, GE, or "other." Attribution of episodes to physicians can be based on different criteria. If episode construction and attribution is used to compare costs across physicians (i.e., physician profiling), cost may be the assignment criterion. For example, an episode may be attributed to the physician with the highest proportion of episode-level costs, or to one or more physicians associated with a minimum proportion of episode-level costs [18, 19]. Episodes may also be attributed to specific providers based on number of physician visits or other measures of utilization [19]. The utilization measures counted toward physician attribution will vary depending on the objective of the assignment, e.g., evaluation and management visits may be used to identify the provider who is primarily responsible for patient care [19]. For this study, episode attribution was intended to identify the physician specialty principally responsible for the management of the subject's GERD during that episode. Therefore, utilization measures were used in order to avoid potential misattribution based on relative cost differences between PCPs and GEs. Episodes were categorized as PCP if at least 55\% of GERD-related utilization, including office visits, procedures, and GERD medication fills, was associated with a PCP physician specialty code (general practitioner, family practitioner or internal medicine). Episodes were classified as GE if at least $55 \%$ of GERD-related utilization was associated with a GE specialty code. All remaining episodes were classified as "other;" these episodes were characterized by specialty codes associated with ear, nose, and throat (ENT), emergency medicine, general surgery, and facilities. The 55\% 
threshold allowed for a clear majority of care between PCPs and GEs while assigning as many episodes to PCPs and GEs as possible.

\section{Medical Chart Abstraction}

Medical charts for 100 subjects were abstracted. The medical chart abstraction was designed to look specifically at the subset of subjects who began GERD-related care with a PCP-consistent with the way patients commonly present with GERD symptoms based on the literature-and were transferred from PCPs to GEs and then back to PCPs. Criteria for subject selection were: at least two episodes; first episode was a PCP episode; evidence of services provided by a GE during a PCP episode or a GE episode subsequent to first PCP episode; a PCP episode following the GE episode (for subjects with a GE episode after the first PCP episode). Thus, based on the claims, all subjects received GERD-related care from both PCPs and GEs in the order PCP-GE-PCP. The last criterion was availability of both PCP and GE charts for abstraction. Two charts, one PCP and one GE, were abstracted for each of the 100 subjects. Information was abstracted from the medical charts for the dates between subjects' first and last observed episodes, and included: reasons that subjects were transferred from the PCP to the GE; reasons for GERD therapy regimen change; GERD-related symptoms; and the circumstances of subjects' return to PCPs after receiving care from GEs.

\section{Analysis}

Claims- and survey-based variables were analyzed descriptively. GERD-related utilization within episodes was compared between PCP and GE episodes. $T$-test and chi-square test were used to identify significant differences in unadjusted means and proportions, respectively.

Logistic regressions were estimated to identify the determinants of subject transfer from PCP to GE, and for transfer from GE to PCP. To model PCP-to-GE transfer, observations for subjects with at least two episodes and a first PCP episode were included in the analysis. The binary, discrete, dependent variable measured whether the subject had at least one GE episode after the first PCP episode $(=1)$. To model the GE-to-PCP transfer, observations for subjects with multiple episodes and a first GE episode were analyzed. The binary, discrete, dependent variable for this model indicated whether the subject had at least one PCP episode after the first GE episode $(=1)$. Independent variables included new-onset GERD subject indicator, age, gender, GERD severity, selected comorbid conditions, use of a PPI, H2RA, or other GERD agent during the first episode, indicators of esophagitis, esophageal stricture, esophageal ulcer, and Barrett's esophagus during the first episode, and an indicator for an endoscopic procedure during the first episode (GE-to-PCP regression only). Regression results are presented as odds ratios (OR) with 95\% confidence intervals $(\mathrm{CI})$.

\section{Results}

\section{Subject Characteristics}

A total of 335,786 subjects were identified based on the claims-based selection criteria. Of those subjects, 270,325 $(80.5 \%)$ had at least one episode assigned to one of the four predetermined GERD-related care categories. A total of 425,404 episodes were identified, for an average of 1.6 episodes per subject. Fifty-four percent $(N=227,719)$ of the episodes were PCP, 46,241 (11\%) were GE, and $150,444(35 \%)$ were other. The sample of subjects included in the analysis was limited to those with a first episode associated with a PCP or GE, yielding 169,884 subjects with 251,347 episodes among them: 211,043 PCP episodes $(84.0 \%)$ and 40,304 GE episodes (16.0\%).

Table 1 provides demographic and pre-index date health status characteristics by first-episode group. Of clinical relevance, subjects whose first episode was GE were significantly more likely to be classified with medium or high GERD severity (26.23\% compared with $16.81 \%$ of subjects with a first PCP episode, $P<0.001)$ and other gastrointestinal disorders (12.40\% compared with $7.14 \%$ of subjects with a first PCP episode, $P<0.001)$. Subjects with a first GE episode also were significantly more likely $(P<0.001)$ to have a diagnosis of esophageal ulcer $(6.54 \%$ compared with $0.63 \%$ for subjects with a first PCP episode), esophagitis (15.34\% compared with $3.19 \%)$, esophageal stricture (5.81\% compared with $0.59 \%)$, and Barrett's esophagus (2.07\% compared with $0.20 \%$ ) during their first episodes.

\section{Health Care Resource Utilization}

The distributions of GERD therapy strategies for all PCP episodes and GE episodes are shown in Fig. 1. The therapies represented in the PCP and GE episode categories were based on pharmacy claims associated with PCP and GE specialty codes, respectively (i.e., GERD therapies prescribed by PCPs in PCP episodes and by GEs in GE episodes). ${ }^{3}$ Some episodes, $13.7 \%$ of PCP episodes and

\footnotetext{
3 The vast majority of GERD therapy prescriptions in PCP episodes were associated with PCPs: $98.3 \%$ of PPI pharmacy claims, $96.8 \%$ of H2RA claims, and $85.0 \%$ of other GERD therapy claims. Similarly, $95.9 \%$ of PPI claims, $80.4 \%$ of H2RA claims, and $86.6 \%$ of other GERD therapy claims in GE episodes were associated with GEs.
} 
Table 1 Subject characteristics by first episode
$P C P$ primary care provider, $G E$ gastroenterologist

a These conditions were selected based on prevalence and clinical relevance

${ }^{\mathrm{b}}$ Evidence is defined as $\geq 1$ medical claim with diagnosis for the designated condition in the first or second position

Fig. 1 GERD therapies in PCP and GE episodes. $P C P$ primary care physician, $G E$ gastroenterologist, GERD gastroesophageal reflux disease, $P P I$ proton pump inhibitor, $H 2 R A$ H2-receptor antagonist

\begin{tabular}{|c|c|c|c|c|c|}
\hline & \multicolumn{2}{|c|}{$\begin{array}{l}\text { First episode, PCP } \\
(N=142,394)\end{array}$} & \multicolumn{2}{|c|}{$\begin{array}{l}\text { First episode, GE } \\
(N=27,490)\end{array}$} & \multirow[t]{2}{*}{$P$-value } \\
\hline & $N$ & $\%$ & $N$ & $\%$ & \\
\hline \multicolumn{6}{|l|}{ Gender } \\
\hline Male & 66,527 & 46.72 & 12,542 & 45.62 & \multirow[t]{2}{*}{0.001} \\
\hline Female & 75,867 & 53.28 & 14,948 & 54.38 & \\
\hline \multicolumn{6}{|l|}{ Pre-index GERD severity } \\
\hline Low & 118,447 & 83.18 & 20,280 & 73.77 & \multirow[t]{3}{*}{$<0.001$} \\
\hline Medium & 23,344 & 16.39 & 6,946 & 25.27 & \\
\hline High & 603 & 0.42 & 264 & 0.96 & \\
\hline \multicolumn{6}{|l|}{ Baseline comorbid conditions ${ }^{a}$} \\
\hline Respiratory infections & 33,543 & 23.56 & 7,039 & 25.61 & $<0.001$ \\
\hline Hypertension & 30,154 & 21.18 & 5,149 & 18.73 & $<0.001$ \\
\hline Disorders of lipid metabolism & 29,027 & 20.38 & 5,920 & 21.54 & $<0.001$ \\
\hline Diseases of the heart & 24,715 & 17.36 & 5,182 & 18.85 & $<0.001$ \\
\hline Upper respiratory disease & 17,541 & 12.32 & 4,248 & 15.45 & $<0.001$ \\
\hline Other lower respiratory disease & 16,498 & 11.59 & 3,624 & 13.18 & $<0.001$ \\
\hline Other gastrointestinal disorders & 10,169 & 7.14 & 3,410 & 12.40 & $<0.001$ \\
\hline \multicolumn{6}{|c|}{ Evidence of complications during first episode ${ }^{b}$} \\
\hline Ulcer & 904 & 0.63 & 1,798 & 6.54 & $<0.001$ \\
\hline Esophagitis & 4,541 & 3.19 & 4,216 & 15.34 & $<0.001$ \\
\hline Esophageal stricture & 838 & 0.59 & 1,598 & 5.81 & $<0.001$ \\
\hline Barrett's esophagus & 278 & 0.20 & 568 & 2.07 & $<0.001$ \\
\hline
\end{tabular}

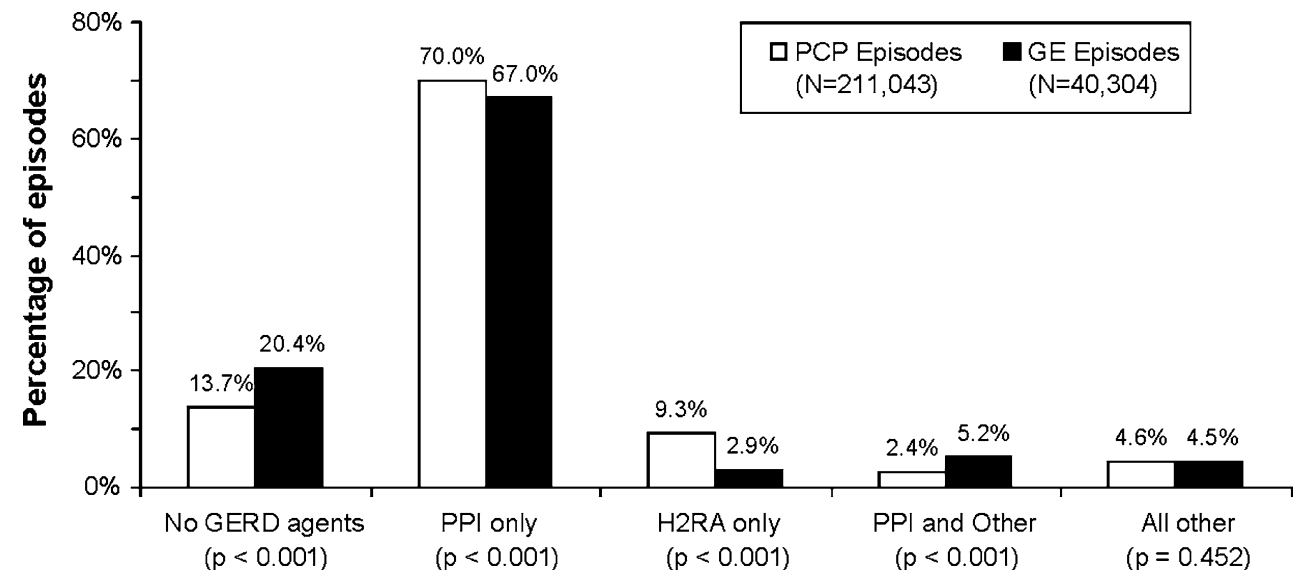

$20.4 \%$ of GE episodes $(P<0.001)$, did not include any pharmacy claims for GERD therapies. PPIs were the sole and predominant therapy in both types of episodes $(70.0 \%$ of PCP episodes, $67.0 \%$ of GE episodes, $P<0.001)$. PCPs were more likely to prescribe only H2RAs (9.3\% of PCP episodes) compared with GEs (2.9\% of GE episodes, $P<0.001)$.

In general, GE episodes included higher levels of unadjusted mean GERD-related utilization rates than did PCP episodes, as shown in Table 2. GE episodes had significantly $(P<0.001)$ higher rates per 100 of GERDrelated physician office visits (119.9 per 100 episodes versus 76.9 per 100 PCP episodes), endoscopic procedures
(50.5/100 versus 6.3/100 PCP episodes), repair and manipulation procedures (3.0/100 versus $0.3 / 100$ PCP episodes), and additional diagnostic procedures (3.2/100 versus $0.3 / 100$ PCP episodes). Not surprisingly, PCP episodes were characterized by significantly higher rates of office visits to PCPs, while GE episodes had higher rates of GE-specific office visits.

Determinants of Transfers and Changes in GERD Therapy

Logistic regression results are provided in Table 3. Esophagitis and esophageal stricture were associated with a 
Table 2 GERD-related health care utilization (unadjusted)

$P C P$ primary care physician, $G E$ gastroenterologist, $S D$ standard deviation

a All variables are rates per 100 episodes

Table 3 Determinants of transfer: logistic regression results

$P C P$ primary care physician, $G E$ gastroenterologist, $P P I$ proton pump inhibitor, $H 2 R A$ $\mathrm{H} 2$-receptor antagonist

* $0.01 \leq P<0.05$;

** $P<0.01$

\begin{tabular}{llcr}
\hline GERD-related utilization rate $^{\mathrm{a}}$ & $\begin{array}{l}\text { PCP episodes } \\
(N=211,043) \\
\text { Mean (SD) }\end{array}$ & $\begin{array}{l}\text { GE episodes } \\
(N=40,304) \\
\text { Mean (SD) }\end{array}$ & $P$-value \\
\hline Any physician office visit & $76.9(94.2)$ & $119.9(102.1)$ & $<0.001$ \\
$\quad$ PCP office visit & $75.2(89.0)$ & $4.3(22.4)$ & $<0.001$ \\
$\quad 1.2(11.8)$ & $113.9(87.9)$ & $<0.001$ \\
GE office visit & $6.3(33.2)$ & $50.5(85.7)$ & $<0.001$ \\
Endoscopic procedures & $0.3(7.5)$ & $3.0(22.5)$ & $<0.001$ \\
Repair/manipulation procedures & $0.3(7.5)$ & $3.2(24.8)$ & $<0.001$ \\
Diagnostic procedures & & &
\end{tabular}

\begin{tabular}{|c|c|c|c|c|}
\hline & \multicolumn{2}{|c|}{ PCP-to-GE model } & \multicolumn{2}{|c|}{ GE-to-PCP model } \\
\hline & Odds ratio & $95 \% \mathrm{CI}$ & Odds ratio & $95 \% \mathrm{CI}$ \\
\hline New-onset GERD & 1.378 & $(1.263-1.503)^{* *}$ & 1.153 & $(1.039-1.279)^{* *}$ \\
\hline Age & 0.996 & $(0.993-0.999)^{*}$ & 1.012 & $(1.008-1.016)^{* *}$ \\
\hline Male & 0.764 & $(0.703-0.831)^{* *}$ & 0.932 & $(0.846-1.027)$ \\
\hline \multicolumn{5}{|l|}{ Pre-index date health status } \\
\hline GERD severity & 0.942 & $(0.840-1.056)$ & 0.849 & $(0.757-0.951)^{* *}$ \\
\hline Disorders of lipid metabolism & 1.062 & $(0.953-1.183)$ & 1.010 & $(0.894-1.140)$ \\
\hline Hypertension & 0.851 & $(0.760-0.953)^{* *}$ & 1.189 & $(1.048-1.348)^{* *}$ \\
\hline Respiratory infections & 0.962 & $(0.872-1.062)$ & 1.017 & $(0.907-1.140)$ \\
\hline Disease of the heart & 1.052 & $(0.938-1.181)$ & 0.920 & $(0.808-1.046)$ \\
\hline Other lower respiratory disease & 1.040 & $(0.911-1.188)$ & 1.008 & $(0.871-1.166)$ \\
\hline Other gastrointestinal disorders & 1.118 & $(0.949-1.316)$ & 1.131 & $(0.969-1.322)$ \\
\hline Lower gastrointestinal disorders & 1.196 & $(0.929-1.540)$ & 1.169 & $(0.922-1.483)$ \\
\hline Other upper respiratory disease & 1.282 & $(1.142-1.439)^{* *}$ & 0.950 & $(0.831-1.085)$ \\
\hline Any PPI use in first episode & 0.503 & $(0.458-0.552)^{* *}$ & 0.595 & $(0.531-0.667)^{* *}$ \\
\hline Any H2RA use in first episode & 0.665 & $(0.585-0.756)^{* *}$ & 0.820 & $(0.691-0.974)^{*}$ \\
\hline Any other GERD therapy use in first episode & 1.034 & $(0.842-1.271)$ & 0.817 & $(0.679-0.983)^{*}$ \\
\hline Esophagitis in first episode & 1.573 & $(1.299-1.905)^{* *}$ & 0.969 & $(0.836-1.124)$ \\
\hline Esophageal ulcer in first episode & 1.101 & $(0.734-1.651)$ & 0.633 & $(0.520-0.771)^{* *}$ \\
\hline Esophageal stricture in first episode & 1.986 & $(1.380-2.857)^{* *}$ & 0.947 & $(0.774-1.159)$ \\
\hline Barrett's esophagus in first episode & 1.369 & $(0.627-2.990)$ & 0.375 & $(0.235-0.599)^{* *}$ \\
\hline Endoscopy during first GE episode & - & - & 1.322 & $(1.174-1.490)^{* *}$ \\
\hline Observations $(N)$ & 62,704 & & 11,318 & \\
\hline
\end{tabular}

57.3\% (CI: 1.299-1.905) and 98.6\% (CI: 1.380-2.857) higher odds of transfer to a GE, respectively. Subjects with upper respiratory disease were 1.282 times (CI: 1.142 1.439) more likely to be transferred. New-onset GERD subjects were $37.8 \%$ more likely (CI: $1.263-1.503$ ) to be transferred from a PCP to a GE than were subjects with GERD-related care before their index dates. Use of PPIs (OR: 0.503, CI: 0.448-0.552) and H2RAs (OR: 0.665, CI: $0.585-0.756)$ during the first PCP episode were associated with significantly lower odds of transfer to a GE. Age was inversely associated with the odds of transfer from a PCP to GE; that is, younger patients were more likely to be transferred, although the effect was very small.
Many of the results from the GE-to-PCP model complemented the results of the PCP-to-GE model. GERD complications were associated with lower odds of transfer to PCP: significant GERD complications were esophageal ulcer (OR: 0.633, CI: 0.520-0.771) and Barrett's esophagus (OR: 0.375, CI: 0.235-0.599). Higher levels of GERD severity were associated with lower odds of transfer from GE to PCP (OR: 0.849, CI: 0.757-0.951). Endoscopic procedures administered during a first GE episode were associated with $32.2 \%$ higher odds (CI: $1.174-1.490)$ of transfer to a PCP. New-onset GERD subjects were 15.3\% more likely (CI: 1.039-1.279) to be transferred from a GE to a PCP. All classes of GERD therapy were associated 
with lower odds of transfer to a PCP (PPI: OR $=0.595$, $\mathrm{CI}=0.531-0.667 ; \quad \mathrm{H} 2 \mathrm{RA}: \quad \mathrm{OR}=0.820, \quad \mathrm{CI}=0.691-$ 0.974 ; other: $\mathrm{OR}=0.817, \mathrm{CI}=0.679-0.983)$. In this model, age was positively associated with the likelihood of transfer from GE to PCP; with each increasing year of age, the odds of a GE-to-PCP transfer increased by $1.2 \%$.

Additional analysis was undertaken to determine whether the higher prevalence rates of esophageal ulcer, esophagitis, esophageal stricture, and Barrett's esophagus in first GE episodes were biased due to GEs' diagnostic approach; that is, were higher proportions of GE episodes characterized with esophageal ulcer, esophagitis, esophageal stricture, and Barrett's esophagus because GEs were more likely to perform endoscopies and, consequently, diagnose these conditions? To evaluate the possibility of such bias, the prevalence of each of these conditions was compared between first PCP and GE episodes that included endoscopic procedures. The results of these comparisons are as follows: $10.8 \%$ of PCP episodes that included endoscopy, and $14.1 \%$ of GE episodes that included endoscopy, also included esophageal ulcer diagnoses; $32.5 \%$ of PCP episodes and $33.3 \%$ of GE episodes that included endoscopy also included esophagitis diagnoses; $3.2 \%$ of PCP episodes and $4.3 \%$ of GE episodes that included endoscopy also included Barrett's esophagus diagnoses; and $10.0 \%$ of PCP episodes and $13.6 \%$ of GE episodes that included endoscopy also included stricture diagnoses. In addition, the analysis was reversed to compare the rate of endoscopic procedure between first PCP and GE episodes that included each of the four conditions. The results showed that: $75.8 \%$ of PCP episodes and $78.1 \%$ of GE episodes that included esophageal ulcer diagnoses also included endoscopy; $42.3 \%$ of PCP episodes and $78.6 \%$ of GE episodes that included esophagitis diagnoses also included endoscopy; $72.7 \%$ of PCP episodes and $76.1 \%$ of GE episodes that included Barrett's esophagus diagnoses also included endoscopy; and $75.5 \%$ of PCP episodes and $84.9 \%$ of GE episodes that included esophageal stricture diagnoses also included endoscopy.

Table 4 provides the frequency of reasons for subject transfer to GEs for GERD care. The most common reasons for transfer of subjects to GEs were to address escalating or an increasing number of symptoms, or evaluation of such gastroenterologic symptoms as abdominal pain, heartburn, chest pain, regurgitation, and indigestion (69 charts), followed by endoscopy (51 charts), and insufficient response to GERD therapy ( 23 charts).

PPIs were, by far, the most prevalent GERD therapy documented in both the PCP and GE charts, as documented in Table 5. A change in GERD therapy regimen over time was documented in $60 \%$ of the PCP charts. A change in regimen typically meant changing the GERD medication ( $N=53$ charts, $88.3 \%$ of charts with regimen change
Table 4 Reasons for transfers to GEs

\begin{tabular}{|c|c|}
\hline Reason for referral from GE charts ${ }^{\mathrm{a}}$ & $\begin{array}{l}\text { Number }(\%) \text { of } \\
\text { charts }\end{array}$ \\
\hline Endoscopic procedure & $51(51.5)$ \\
\hline $\begin{array}{l}\text { General gastroenterological symptoms (abdominal } \\
\text { or epigastric pain, bloating, gas, diarrhea, } \\
\text { indigestion, regurgitation, heartburn, chest pain) }\end{array}$ & $34(34.3)$ \\
\hline Symptom severity & $29(29.3)$ \\
\hline Patient not responding to GERD therapy & $23(23.2)$ \\
\hline Existing or chronic GERD & $19(19.2)$ \\
\hline Diagnostic procedure & $14(14.1)$ \\
\hline Increasing number of symptoms & $6(6.1)$ \\
\hline
\end{tabular}

GE gastroenterologist

${ }^{\text {a }}$ Multiple reasons for referral could be selected; data from GE charts

documented), followed by a change in frequency of medication administration ( $N=10,16.7 \%$, data not shown). Change in GERD therapy was documented in $42 \mathrm{GE}$ charts; again, the most prevalent change in therapy was a change in the GERD medication ( $N=10$ charts, $23.8 \%$ of charts with regimen change documented at first visit), followed by a change in frequency $(N=3,7.1 \%$, data not shown). Reasons for therapy regimen change over time were abstracted from both PCP and GE charts (Table 5). The most common reasons for therapy regimen change were a lack of improvement in GERD symptoms $(51.7 \%$ of PCP charts, $9.5 \%$ of GE charts) and insufficient subject response to therapy (51.7\% of PCP charts, $26.2 \%$ of GE charts). Nineteen percent of GE charts also documented upper gastrointestinal (GI) endoscopy results as a reason for therapy regimen change.

Eighty-seven (88\%) of the GE charts showed that GEs ordered at least one diagnostic endoscopy or esophagoscopy (data not shown). Forty-six (46.5\%) of the GE charts explicitly documented that the GE transferred the subject back to the PCP (data not shown); circumstances around the return of subjects to the PCPs' care included completion of endoscopic or other procedure (39 charts), decreased symptom severity (4 charts), and improved response to therapy (3 charts).

\section{Discussion}

This was a combined retrospective administrative claims and medical chart review study that examined GERDrelated health care resource utilization, GERD therapy, and reasons for transfer of subjects between PCPs and GEs. The results of the descriptive claims-based analysis, medical chart data analysis, and multivariate regression analysis were highly complementary and consistent. 
Table 5 GERD therapy regimens documented in PCP and GE medical charts

\begin{tabular}{|c|c|c|}
\hline & $\begin{array}{l}\text { PCP } N=100 \text { charts } \\
N(\%)\end{array}$ & $\begin{array}{l}\text { GE } N=99 \text { charts }^{\mathrm{a}} \\
N(\%)\end{array}$ \\
\hline Documentation of antacid use & $17(17.0 \%)$ & $7(7.1 \%)$ \\
\hline Documentation of PPI use & $88(88.0 \%)$ & $84(84.8 \%)$ \\
\hline Documentation of H2RA use & $26(26.0 \%)$ & $14(14.1 \%)$ \\
\hline \multicolumn{3}{|l|}{ Reasons for change in GERD therapy regimen over time } \\
\hline Any change in GERD therapy regimen over time & $60(60.0 \%)$ & $42(42.4 \%)$ \\
\hline Symptoms did not change or worsened & $31(51.7 \%)^{\mathrm{b}}$ & $4(9.5 \%)^{\mathrm{c}}$ \\
\hline Patient did not respond to therapy & $31(51.7 \%)^{\mathrm{b}}$ & $11(26.2 \%)^{\mathrm{c}}$ \\
\hline Change in insurance coverage & $4(6.7 \%)^{\mathrm{b}}$ & $1(2.4 \%)^{\mathrm{c}}$ \\
\hline Patient request & $2(3.3 \%)^{\mathrm{b}}$ & 0 \\
\hline Symptoms improved & 0 & $3(7.1 \%)^{\mathrm{c}}$ \\
\hline EGD results & 0 & $8(19.0 \%)^{\mathrm{c}}$ \\
\hline Other reason & $6(10.0 \%)^{\mathrm{b}}$ & $6(14.3 \%)^{\mathrm{c}}$ \\
\hline No information in chart & $9(15.0 \%)^{\mathrm{b}}$ & $13(31.0 \%)^{\mathrm{c}}$ \\
\hline
\end{tabular}

$P C P$ primary care physician, $G E$ gastroenterologist, $P P I$ proton pump inhibitor, H2RA H2-receptor antagonist, EGD esophagogastroduodenoscopy

${ }^{a}$ One GE chart had no information about the subject during the designated time period, thus data from 99 GE charts were analyzed

${ }^{b}$ Percentages are proportions of 60 PCP charts with documentation of change in GERD therapy regimen over time

${ }^{c}$ Percentages are proportions of 42 GE charts with documentation of change in GERD therapy regimen over time; multiple responses could be selected

PPIs were the principal GERD therapy, followed by H2RA. PPIs were associated with significantly lower likelihood of transfer both from PCP to GE and from GE to PCP in the multivariate analysis, suggesting that PPI use is so prevalent that it is unlikely to influence transfer decisions.

GE episodes, in general, were characterized by higher rates of physician office visits, endoscopic procedures, esophageal repair and manipulation procedures, and additional GERD diagnostic procedures. Subjects who were transferred to GEs were more likely to have endoscopies than were subjects who were not transferred. Subjects whose first episodes were GE episodes tended to have higher GERD severity levels and higher rates of baseline comorbid conditions. Although the differences in the rates of baseline comorbid conditions were statistically significant, they do not represent clinically significant differences. Subjects whose first episodes were GE were more likely to have general GI disorders, esophageal ulcer, esophagitis, esophageal stricture, and Barrett's esophagus. Moreover, subjects with relatively severe GERD symptoms or complications tended to be transferred to, or remain under the care of, GEs; it is possible that the coefficient for Barrett's esophagus in the PCP-to-GE transfer regression was not significant because a very small proportion of subjects in first PCP episodes had a diagnosis of Barrett's esophagus.

Small proportions of subjects whose first episodes were PCP had diagnoses of Barrett's esophagus or esophagitis or esophageal stricture. We speculate that most of these subjects were diagnosed with these conditions prior to our study period, and were managed by PCPs. Moreover, it is important to bear in mind how episodes were assigned to PCP or GE, i.e., based on at least 55\% of GERD-related utilization. It is therefore possible that subjects with these conditions who were under the care of PCPs could still have been seen by GEs (and have even undergone endoscopy) during their PCP episodes.

The additional analysis that compared the prevalence of these conditions between PCP and GE episodes with endoscopy mitigates the likelihood that the result that GEs treat more severe GERD patients was biased by the higher rate of endoscopic procedures among GEs.

Together, these results indicate that, in general, PCPs treat GERD patients with mild symptoms and symptoms that respond to initial GERD pharmaceutical therapy, while patients whose symptoms do not respond to standard GERD therapy, or whose symptoms are severe when they begin care, are treated by GEs. These results also indicate that PCPs empirically treat patients with GERD, usually with a step-down approach, while GEs tend to adopt a more resource-intensive treatment approach, including greater numbers of office visits and interventions.

There are limitations inherent to administrative claims, which are collected for the purpose of payment and not for research. A pharmacy claim for a filled prescription does not indicate that the medication was consumed or that it was taken as prescribed. OTC medications or those 
provided as samples by the physician are not observed in the claims data; this qualification might have led to an underestimate of H2RA use within the study population. A diagnosis code on a medical claim is not necessarily positive presence of condition, as the diagnosis code may have been coded incorrectly or included as a rule-out criterion. For this study, however, subjects had to meet multiple clinical criteria, making inclusion based on rule-out unlikely.

For this study specifically, there were additional limitations. First, results are based primarily on subjects' claims experience during the study period. Previous treatment could not be observed, but likely contributed to the rate of health care resource utilization during the first and subsequent treatment episodes. Furthermore, the absence of clinical information in claims data does not allow us to verify the inferences made with respect to physicians' behavior and clinical motivations. Second, results from the medical chart abstraction data should be applied to larger populations cautiously; generalizability is limited. Third, utilization of OTC medications for treatment of GERD symptoms is an important component of care to understand and quantify. It was, unfortunately, difficult to know the extent to which the rates of OTC antacid and H2RA use reflected in the chart abstraction results are accurate or generalizable among individuals with GERD. Fourth, the criteria for assigning episodes to PCPs and GEs was made purposefully, but may limit the generalizability of these results; the alignment of the claims analysis results from this study with those from survey analyses published in the literature, as well as with those from the medical chart abstraction, mitigates this limitation. Finally, integrating information about "other" episodes was beyond the scope of this study. Additional analysis suggested that other episodes often included active participation by both PCPs and GEs; thus, some information about PCP and GE behaviors was excluded from this analysis.

This study provides a real-world examination of PCP and GE treatment of GERD in a large, national population. It provides important breadth to published physician-based survey data on GERD treatment. The data showed that PCPs tend to treat relatively uncomplicated cases of GERD; their treatment approach appears to be empirical and step-down, based on heavy PPI utilization. PCPs transfer GERD patients to the care of GEs when the patients do not respond to therapy and when their symptoms grow more severe. GEs tend to adopt a diagnostic treatment approach with endoscopic procedures, and transfer the care of the patient back to the PCP when GERD symptoms are diagnosed with endoscopy or when symptoms have improved. Based on these data, we speculate that timely transfers of patients with GERD symptoms from PCPs to GEs when empiric treatment appears to be insufficient may lead to more effective and efficient management of GERD.

Acknowledgments The authors would like to thank Thomas E. Lynn, MD of Ingenix for his invaluable assistance with the Episode Treatment Group ${ }^{\mathrm{TM}}$ software, and to acknowledge support provided by TAP Pharmaceutical Products, Inc., Lake Forest, IL, which is now part of Takeda Pharmaceuticals North America, Inc. (TPNA), Deerfield, IL. i3 Innovus received financial support from TPNA as part of this research contract.

\section{References}

1. Sontag SJ. The medical management of reflux esophagitis. Role of antacids and acid inhibition. Gastroenterol Clin North Am. 1990;19:683-712.

2. Howard PJ, Heading RC. Epidemiology of gastro-esophageal reflux disease. World J Surg. 1992;16:288-293. doi:10.1007/ BF02071535.

3. Hinder RA, Libbey JS, Gorecki P, Bammer T. Antireflux surgery: indications, preoperative evaluation, and outcome. Gastroenterol Clin North Am. 1999;28:987-1005. doi:10.1016/S0889-8553(05) 70101-1. viii.

4. Richter JE. The many manifestations of gastroesophageal reflux disease: presentation, evaluation, and treatment. Gastroenterol Clin North Am. 2007;36:577-599. doi:10.1016/j.gtc.2007.07.014. Viii-ix.

5. Van Pinxteren B, Numans ME, Bonis PA, Lau J. Short-term treatment with proton pump inhibitors, H2-receptor antagonists and prokinetics for gastrooesophageal reflux disease-like symptoms and endoscopy negative reflux disease. Cochrane Database Syst Rev. 2001;(4):CD002095.

6. DeVault KR, Castell DO. American College of Gastroenterology: updated guidelines for the diagnosis and treatment of gastroesophageal reflux disease. Am J Gastroenterol. 2005;100:190200. doi:10.1111/j.1572-0241.2005.41217.x.

7. Voutilainen M, Sipponen P, Mecklin JP, Juhola M, Farkkila M. Gastroesophageal reflux disease: prevalence, clinical, endoscopic and histopathological findings in 1,128 consecutive patients referred for endoscopy due to dyspeptic and reflux symptoms. Digestion. 2000;61:6-13. doi:10.1159/000007730.

8. Chey WD, Inadomi JM, Booher AM, Sharma VK, Fendrick AM, Howden CW. Primary-care physicians' perceptions and practices on the management of GERD: results of a national survey. Am J Gastroenterol. 2005;100:1237-1242. doi:10.1111/j.1572-0241. 2005.41364.x.

9. Castell DO, Brunton SA, Earnest DL, et al. GERD: management algorithms for the primary care physician and the specialist. Pract Gastroenterol. 1999;23:20-44.

10. Dent $J$. The role of the specialist in the diagnosis and short and long term care of patients with gastroesophageal reflux disease. Am J Gastroenterol. 2001;96(8 Suppl):S22-S26. doi:10.1016/ S0002-9270(01)02578-3.

11. Wilcox CM, Heudebert G, Klapow J, Shewchuck R, Casebeer L. Survey of primary care physicians' approach to gastroesophageal reflux disease in elderly patients. J Gerontol A Biol Sci Med Sci. 2001;56:M514-M517.

12. Meining A, Driesnack U, Classen M, Rosch T. Management of gastroesophageal reflux disease in primary care: results of a survey in 2 areas in Germany. Z Gastroenterol. 2002;40:15-20. doi:10.1055/s-2002-19638.

13. Boolchand V, Faulx A, Das A, et al. Primary care physician attitudes toward endoscopic screening for GERD symptoms and 
unsedated esophagoscopy. Gastrointest Endosc. 2006;63:228233. doi:10.1016/j.gie.2005.06.044.

14. Wong WM, Lim P, Wong BC. Clinical practice patterns of gastroenterologists, primary care physicians, and otolaryngologists for the management of GERD in the Asia-Pacific region: the FAST survey. J Gastroenterol Hepatol. 2004;19(Suppl 3): S54-S60. doi:10.1111/j.1440-1746.2004.03590.x.

15. Lacy BE, Crowell MD, Riesett RP, Mitchell A. Age, specialty, and practice setting predict gastroesophageal reflux disease prescribing behavior. J Clin Gastroenterol. 2005;39:489-494. doi: 10.1097/01.mcg.0000165647.24748.04.

16. Episode Treatment Groups ${ }^{\mathrm{TM}}$ (ETG). Release 6.0, 2006. Software documentation obtained from Symmetry, an Ingenix Company.
17. Comorbidity software. Version 3.2. Available at: http://hcup-us. ahrq.gov/toolssoftware/comorbidity/comorbidity.jsp. Accessed 6 Feb 2009.

18. Thomas JW, Ward K. Economic profiling of physician specialists: use of outlier treatment and episode attribution rules. Inquiry. 2006;43:271-282.

19. Medicare Payment Advisory Commission. Report to Congress: increasing the value of medicare. Chap. 1: using episode groupers to assess physician use; 2006. Available at www.medpac.gov. Accessed 30 July 2009. 